\title{
An Erdös-Révész Type Law of the Iterated Logarithm for Order Statistics of a Stationary Gaussian Process
}

\author{
K. Dębicki ${ }^{1}$ • K. M. Kosiński ${ }^{1}$
}

Received: 10 June 2016 / Revised: 10 July 2016 / Published online: 7 September 2016 (C) The Author(s) 2016. This article is published with open access at Springerlink.com

Abstract Let $\left\{X(t): t \in \mathbb{R}_{+}\right\}$be a stationary Gaussian process with almost surely (a.s.) continuous sample paths, $\mathbb{E} X(t)=0, \mathbb{E} X^{2}(t)=1$ and correlation function satisfying (i) $r(t)=1-C|t|^{\alpha}+o\left(|t|^{\alpha}\right)$ as $t \rightarrow 0$ for some $0 \leq \alpha \leq 2$ and $C>0$; (ii) $\sup _{t \geq s}|r(t)|<1$ for each $s>0$ and (iii) $r(t)=O\left(t^{-\lambda}\right)$ as $t \rightarrow \infty$ for some $\lambda>0$. For any $n \geq 1$, consider $n$ mutually independent copies of $X$ and denote by $\left\{X_{r: n}(t)\right.$ : $t \geq 0\}$ the $r$ th smallest order statistics process, $1 \leq r \leq n$. We provide a tractable criterion for assessing whether, for any positive, non-decreasing function $f, \mathbb{P}\left(\mathscr{E}_{f}\right)=$ $\mathbb{P}\left(X_{r: n}(t)>f(t)\right.$ i.o. $)$ equals 0 or 1 . Using this criterion we find, for a family of functions $f_{p}(t)$ such that $z_{p}(t)=\mathbb{P}\left(\sup _{s \in[0,1]} X_{r: n}(s)>f_{p}(t)\right)=O\left(\left(t \log ^{1-p} t\right)^{-1}\right)$, that $\mathbb{P}\left(\mathscr{E}_{f_{p}}\right)=1_{\{p \geq 0\}}$. Consequently, with $\xi_{p}(t)=\sup \left\{s: 0 \leq s \leq t, X_{r: n}(s) \geq f_{p}(s)\right\}$, for $p \geq 0$ we have $\lim _{t \rightarrow \infty} \xi_{p}(t)=\infty$ and $\lim \sup _{t \rightarrow \infty}\left(\xi_{p}(t)-t\right)=0$ a.s. Complementarily, we prove an Erdös-Révész type law of the iterated logarithm lower bound on $\xi_{p}(t)$, namely, that $\liminf _{t \rightarrow \infty}\left(\xi_{p}(t)-t\right) / h_{p}(t)=-1$ a.s. for $p>1$ and $\liminf _{t \rightarrow \infty} \log \left(\xi_{p}(t) / t\right) /\left(h_{p}(t) / t\right)=-1$ a.s. for $p \in(0,1]$, where $h_{p}(t)=\left(1 / z_{p}(t)\right) p \log \log t$.

Keywords Extremes of Gaussian processes - Order statistics process - Law of the iterated logarithm

Mathematics Subject Classification (2010) Primary: 60F15, 60G70 - Secondary: $60 \mathrm{G} 22$

$\triangle$ K. M. Kosiński

Kamil.Kosinski@math.uni.wroc.pl

K. Dębicki

Krzysztof.Debicki@math.uni.wroc.pl

1 Instytut Matematyczny, University of Wrocław, Pl. Grunwaldzki 2/4, 50-384 Wrocław, Poland 


\section{Introduction and Main Results}

Let $X=\left\{X(t): t \in \mathbb{R}_{+}\right\}$be a stationary Gaussian process with almost surely (a.s.) continuous sample paths, $\mathbb{E} X(t)=0$ and $\mathbb{E} X^{2}(t)=1$. Suppose that the correlation function of $X, r(t)=\mathbb{E} X(t) X(0)$, satisfies the following regularity assumptions:

$$
\begin{aligned}
r(t) & =1-C|t|^{\alpha}+o\left(|t|^{\alpha}\right) \quad \text { as } t \rightarrow 0 \text { for some } 0 \leq \alpha \leq 2, \quad C>0, \\
r^{*}(s) & =\sup _{t \geq s}|r(t)|<1 \quad \text { for each } s>0, \\
r(t) & =O\left(t^{-2 \lambda}\right) \text { as } t \rightarrow \infty \text { for some } \lambda>0 .
\end{aligned}
$$

The analysis of extremes of Gaussian stochastic processes has a long history. The celebrated double sum method, primarily developed by Pickands, e.g., [8], and extended by seminal works of Piterbarg, e.g., [10] or monograph [9], plays central role in the extreme value theory of Gaussian processes. The technique developed there appeared to be an universal method, which may deliver answers also to classes of non-Gaussian processes, see for example, recent contributions of $[5,6]$.

Laws of the iterated logarithm take important place in this theory, providing properties of extremal behavior of stochastic processes on large-time scale. One of important contributions in this domain is a result on the process $\xi=\{\xi(t): t \geq 0\}$, defined via $\xi(t)=\sup \left\{s: 0 \leq s \leq t, X(s) \geq(2 \log s)^{1 / 2}\right\}$. In particular, the law of the iterated logarithm implies that, see $[11,12]$,

$$
\limsup _{t \rightarrow \infty}(\xi(t)-t)=0 \text { a.s. }
$$

Interestingly, under the above regularity assumptions, [12] gave the lower bound of $\xi(t)$ and obtained an Erdös-Révész type law of the iterated logarithm, that is,

$$
\begin{aligned}
\liminf _{t \rightarrow \infty} \frac{\xi(t)-t}{t(\log t)^{(\alpha-2) /(2 \alpha) \cdot \log _{2} t}} & =-\frac{(2+\alpha) \sqrt{\pi}}{\alpha \mathcal{H}_{\alpha}(2 C)^{1 / \alpha}} \quad \text { a.s. if } 0<\alpha<2, \\
\liminf _{t \rightarrow \infty} \frac{\log (\xi(t) / t)}{\log _{2} t} & =-\frac{2 \sqrt{\pi}}{\mathcal{H}_{2} \sqrt{2 C}} \quad \text { a.s. if } \alpha=2,
\end{aligned}
$$

where $\mathcal{H}_{\alpha}$ is the Pickands' constant defined by $\mathcal{H}_{\alpha}=\lim _{T \rightarrow \infty} T^{-1} \mathbb{E}$ $e^{\sup _{t \in[0, T]}\left(\sqrt{2} B_{\alpha / 2}(t)-t^{\alpha}\right)}$, with $B_{\alpha / 2}=\left\{B_{\alpha / 2}(t): t \geq 0\right\}$ denoting fractional Brownian motion with Hurst index $\alpha / 2 \in(0,1]$, i.e., a continuous, centered Gaussian process with covariance function

$$
\mathbb{E} B_{\alpha / 2}(s) B_{\alpha / 2}(t)=\frac{1}{2}\left(|s|^{\alpha}+|t|^{\alpha}-|t-s|^{\alpha}\right) .
$$

Equation (3) shows that for any $t$ big enough there exists an $s$ in $\left[t-t(\log t)^{(\alpha-2) /(2 \alpha)}\right.$. $\left.\log _{2} t, t\right]$ such that, almost surely, $X(s) \geq(2 \log s)^{1 / 2}$ and that the length of the interval $t(\log t)^{(\alpha-2) /(2 \alpha)} \cdot \log _{2} t$ is smallest possible. Moreover, the bigger the parameter $\alpha$ is, the wider the interval will be. 
In this paper, we derive a counterpart of Shao's result for the order statistics process $X_{r: n}$. Namely, for any $n \geq 0$, we consider $X_{1}, \ldots, X_{n}, n$ mutually independent copies of $X$ and denote by $X_{r: n}=\left\{X_{r: n}(t): t \geq 0\right\}$ the $r$ th smallest order statistics process, that is, for each $t \geq 0,1 \leq r \leq n$,

$$
X_{1: n}(t)=\min _{1 \leq j \leq n} X_{j}(t) \leq X_{2: n}(t) \leq \ldots \leq X_{n-1: n}(t) \leq \max _{1 \leq j \leq n} X_{j}(t)=X_{n: n}(t) .
$$

Our first contribution is the theorem that extends classical findings of Qualls and Watanabe [11].

Theorem 1 For all functions $f$ that are positive and non-decreasing on some interval $[T, \infty), T>0$, it follows that

$$
\mathbb{P}\left(\mathscr{E}_{f}\right):=\mathbb{P}\left(X_{r: n}(t)>f(t) \text { i.o. }\right)=0 \text { or } 1
$$

as the integral

$$
\mathscr{I}_{f}:=\int_{T}^{\infty} \mathbb{P}\left(\sup _{t \in[0,1]} X_{r: n}(t)>f(u)\right) \mathrm{d} u \text { is finite or infinite. }
$$

[1, Theorem 2.2], see also [3], gave the expression for the asymptotic behavior of the probability in $\mathscr{I}_{f}$, namely

$$
\mathbb{P}\left(\sup _{t \in[0,1]} X_{r: n}(t)>u\right)=C^{\frac{1}{\alpha}}\left(\begin{array}{l}
n \\
\hat{r}
\end{array}\right) \mathcal{H}_{\alpha, \hat{r}} u^{\frac{2}{\alpha}}(\Psi(u))^{\hat{r}}(1+o(1)), \quad \text { as } u \rightarrow \infty
$$

where $\hat{r}=n-r+1, \Psi(u)=1-\Phi(u)$ and $\Phi(u)$ is the distribution function of unit normal law,

$$
\begin{aligned}
\mathcal{H}_{\alpha, k} & =\lim _{T \rightarrow \infty} T^{-1} \mathcal{H}_{\alpha, k}(T) \in(0, \infty), \\
\mathcal{H}_{\alpha, k}(T) & =\int_{\mathbb{R}^{k}} e^{\sum_{i=1}^{k} w_{i}} \mathbb{P}\left(\sup _{t \in[0, T]} \min _{1 \leq i \leq k}\left(\sqrt{2} B_{\alpha / 2}^{(i)}(t)-t^{\alpha}-w_{i}\right)>0\right) \mathrm{d} w_{1} \ldots \mathrm{d} w_{k}
\end{aligned}
$$

and $B_{\alpha / 2}^{(i)}, 1 \leq i \leq n$, are mutually independent fractional Brownian motions. $\mathcal{H}_{\alpha, k}$ is the generalized Pickands' constant introduced in [2]; see also [1]. Therefore, Theorem 1 provides a tractable criterion for settling the dichotomy of $\mathbb{P}\left(\mathscr{E}_{f}\right)$.

For instance, let

$$
f_{p}(s)=\left(\frac{2}{\hat{r}}\left(\log s+\left(\frac{2-\hat{r} \alpha}{2 \alpha}+1-p\right) \log _{2} s\right)\right)^{\frac{1}{2}}, \quad p \in \mathbb{R}
$$


One easily checks that, as $u \rightarrow \infty$,

$$
\mathbb{P}\left(\sup _{t \in[0,1]} X_{r: n}(t)>f_{p}(u)\right)=C^{\frac{1}{\alpha}}\left(\begin{array}{l}
n \\
\hat{r}
\end{array}\right) \frac{\mathcal{H}_{\alpha, \hat{r}}}{(2 \pi)^{\frac{\hat{r}}{2}}}\left(\frac{2}{\hat{r}}\right)^{\frac{2-\hat{r} \alpha}{2 \alpha}}\left(u \log ^{1-p} u\right)^{-1}(1+o(1)) .
$$

Hence, for any $p \in \mathbb{R}$,

$$
\mathbb{P}\left(X_{r: n}(t)>f_{p}(t) \text { i.o. }\right)=\left\{\begin{array}{l}
1 \text { if } p \geq 0 \\
0 \text { if } p<0
\end{array} .\right.
$$

Furthermore,

$$
\limsup _{t \rightarrow \infty} \frac{X_{r: n}(t)}{\sqrt{\log t}}=\sqrt{\frac{2}{\hat{r}}} \text { a.s. }
$$

Next, consider the process $\xi_{p}=\left\{\xi_{p}(t): t \geq 0\right\}$ defined as

$$
\xi_{p}(t)=\sup \left\{s: 0 \leq s \leq t, X_{r: n}(s) \geq f_{p}(s)\right\} .
$$

Since $\mathscr{I}_{f_{p}}=\infty$ for $p \geq 0$, Theorem 1 implies that

$$
\lim _{t \rightarrow \infty} \xi_{p}(t)=\infty \quad \text { a.s. } \text { and } \limsup _{t \rightarrow \infty}\left(\xi_{p}(t)-t\right)=0 \quad \text { a.s. }
$$

Let, cf. (6),

$$
h_{p}(t)=p\left(\mathbb{P}\left(\sup _{s \in[0,1]} X_{r: n}(s)>f_{p}(t)\right)\right)^{-1} \log _{2} t
$$

The second contribution of this paper is an Erdös-Révśz type of law of the iterated logarithm for the process $\xi_{p}$.

Theorem 2 If $p>1$, then

$$
\liminf _{t \rightarrow \infty} \frac{\xi_{p}(t)-t}{h_{p}(t)}=-1 \text { a.s. }
$$

If $p \in(0,1]$, then

$$
\liminf _{t \rightarrow \infty} \frac{\log \left(\xi_{p}(t) / t\right)}{h_{p}(t) / t}=-1 \text { a.s. }
$$

Now, let us complementary put $\eta_{p}=\left\{\eta_{p}(t): t \geq 0\right\}$, where

$$
\eta_{p}(t)=\inf \left\{s \geq t: X_{r: n}(s) \geq f_{p}(s)\right\}
$$


Since

$$
\mathbb{P}\left(\xi_{p}(t)-t \leq-x\right)=\mathbb{P}\left(\sup _{s \in(t-x, t]} \frac{X_{r: n}(s)}{f_{p}(s)}<1\right)
$$

and

$$
\mathbb{P}\left(z-\eta_{p}(z) \leq-x\right)=\mathbb{P}\left(\sup _{s \in[z, z+x]} \frac{X_{r: n}(s)}{f_{p}(s)}<1\right)
$$

then it follows that

$$
\liminf _{t \rightarrow \infty} \frac{\xi_{p}(t)-t}{h_{p}(t)}=\liminf _{z \rightarrow \infty} \frac{z-\eta_{p}(z)}{h_{p}(z)} .
$$

Theorem 2 shows that for $t$ big enough, there exists an $s$ in $\left[t-h_{p}(t), t\right]$ (as well as in $\left[t, t+h_{p}(t)\right]$ by (7)) such that $X_{r: n}(s) \geq f_{p}(s)$ and that the length of the interval $h_{p}(t)$ is smallest possible. One can retrieve (3)-(4) by setting $n=1$, and $p=\frac{2-\hat{r} \alpha}{2 \alpha}+1=\frac{2+\alpha}{2 \alpha}$. Theorem 2 not only generalizes [12, Theorem 1.1 ], it also unveils the lacking so far structure of the lower bound of $\xi_{p}(t)$ by relating it, via $h_{p}(t)$, to the asymptotics of the tail distribution of the supremum of the underlying process evaluated at $f_{p}(t)$; in (3) $t(\log t)^{(\alpha-2) /(2 \alpha)}$ is of the same asymptotic order as the reciprocal of $\mathbb{P}\left(\sup _{s \in[0,1]} X(s)>(2 \log t)^{1 / 2}\right)$. This shines new light on this type of results, which appear to be intrinsically connected with Gumbel limit theorems; see, e.g., [7], where the function $h_{p}(t)$ plays crucial role. We shall pursue this elsewhere.

The paper is organized as follows. In Sect. 2, we provide a collection of basic results on order statistics of stationary Gaussian processes, used throughout the paper, and prove auxiliary lemmas, which constitute building blocks of the proofs of the main results. These are given in the final part of the paper, Sect. 3.

\section{Auxiliary Lemmas}

We begin with some auxiliary lemmas that are later needed in the proofs.

The following lemma is the general form of the Borel-Cantelli lemma; cf. [13].

Lemma 1 Consider a sequence of events $\left\{E_{k}: k \geq 0\right\}$. If

$$
\sum_{k=0}^{\infty} \mathbb{P}\left(E_{k}\right)<\infty
$$

then $\mathbb{P}\left(E_{n}\right.$ i.o. $)=0$. Whereas, if

$$
\sum_{k=0}^{\infty} \mathbb{P}\left(E_{k}\right)=\infty \text { and } \liminf _{n \rightarrow \infty} \frac{\sum_{1 \leq k \neq t \leq n} \mathbb{P}\left(E_{k} E_{t}\right)}{\left(\sum_{k=1}^{n} \mathbb{P}\left(E_{k}\right)\right)^{2}} \leq 1,
$$

then $\mathbb{P}\left(E_{n}\right.$ i.o. $)=1$. 
The following two lemmas constitute useful tools for approximating the supremum of $X_{r: n}$ on a fixed interval by its maximum on a grid with a sufficiently dense mesh.

Lemma 2 There exist positive constants $K, c$ and $u_{0}$ such that

$$
\begin{aligned}
& \mathbb{P}\left(\max _{0 \leq j \leq u^{\frac{2}{\alpha}} / \theta} X_{r: n}\left(j \theta u^{-\frac{2}{\alpha}}\right) \leq u-\frac{\theta^{\frac{\alpha}{4}}}{u}, \sup _{t \in[0,1]} X_{r: n}(t)>u\right) \\
& \quad \leq K u^{\frac{2 \hat{r}}{\alpha}}(\Psi(u))^{\hat{r}} \theta^{\frac{\alpha}{2}-1} \Psi\left(c \theta^{-\frac{\alpha}{4}}\right),
\end{aligned}
$$

for each $\theta>0$ and $u \geq u_{0}$.

Proof Note that, by stationarity, there exists a constant $K$, that may vary from line to line, such that, for sufficiently large $u$,

$$
\begin{aligned}
& \mathbb{P}\left(\max _{0 \leq j \leq u^{\frac{2}{\alpha}} / \theta} X_{r: n}\left(j \theta u^{-\frac{2}{\alpha}}\right) \leq u-\frac{\theta^{\frac{\alpha}{4}}}{u}, \sup _{t \in[0,1]} X_{r: n}(t)>u\right) \\
& \leq \frac{u^{\frac{2}{\alpha}}}{\theta} \mathbb{P}\left(X_{r: n}(0) \leq u-\frac{\theta^{\frac{\alpha}{4}}}{u}, \sup _{t \in[0,1]} X_{r: n}(t)>u\right) \\
& \leq \frac{u^{\frac{2}{\alpha}}}{\theta}\left(\begin{array}{l}
n \\
r
\end{array}\right)\left(\begin{array}{c}
n \\
n-r+1
\end{array}\right) \mathbb{P}\left(\forall_{i=1, \ldots, r} X_{i}(0) \leq u-\frac{\theta^{\frac{\alpha}{4}}}{u}, \forall_{j=r, \ldots, n} \sup _{t \in[0,1]} X_{j}(t)>u\right) \\
& \leq K \frac{u^{\frac{2}{\alpha}}}{\theta} \mathbb{P}\left(X_{r}(0) \leq u-\frac{\theta^{\frac{\alpha}{4}}}{u}, \sup _{t \in[0,1]} X_{r}(t)>u\right)\left(\mathbb{P}\left(\sup _{t \in[0,1]} X(t)>u\right)\right)^{n-r} \\
& \leq K u^{\frac{2 \hat{r}}{\alpha}}(\Psi(u))^{\hat{r}} \theta^{\frac{\alpha}{2}-1} \Psi\left(c \theta^{-\frac{\alpha}{4}}\right) .
\end{aligned}
$$

The last inequality follows from (5) and the classical result of [7, Lemma 12.2.5], where the constant $c>0$ is given therein.

The proof of the following lemma follows line-by-line the same reasoning as the proof of [1, Theorem 2.2], and thus we omit it.

Lemma 3 For any $\theta>0$, as $u \rightarrow \infty$,

$$
\mathbb{P}\left(\max _{0 \leq j \leq u^{\frac{2}{\alpha}} / \theta} X_{r: n}\left(j \theta u^{-\frac{2}{\alpha}}\right)>u\right)=C^{\frac{1}{\alpha}}\left(\begin{array}{l}
n \\
\hat{r}
\end{array}\right) \frac{\mathcal{H}_{\alpha, \hat{r}}(\theta)}{\theta}(\Psi(u))^{\hat{r}}(1+o(1)) .
$$

The next lemma follows directly from [4, Theorem 2.4] and is a generalization of the classical Berman's inequality to order statistics.

Lemma 4 For some $n, d \geq 1$, and any $1 \leq l \leq n$ let $\left\{\xi_{l}^{(0)}(i): 1 \leq i \leq d\right\}$ and $\left\{\xi_{l}^{(1)}(i): 1 \leq i \leq d\right\}$ be a sequence of $\mathcal{N}(0,1)$ variables and set $\sigma_{i l, j k}^{(\kappa)}=$ $E \xi_{l}^{(\kappa)}(i) \xi_{k}^{(\kappa)}(j), \kappa=0,1$. For any $1 \leq r \leq n$ and $1 \leq i \leq d$, let $\xi_{r: n}^{(\kappa)}(i)$ be the rth 
order statistic of $\xi_{1}^{(\kappa)}(i), \ldots, \xi_{n}^{(\kappa)}(i)$. Suppose that, for any $1 \leq i, j \leq d, 1 \leq l, k \leq$ $n, \kappa=0,1$,

$$
\sigma_{i l, j k}^{(\kappa)}=\sigma_{i j}^{(\kappa)} 1_{\{l=k\}}
$$

for some $\sigma_{i j}^{(\kappa)}$. Now define

$$
\rho_{i j}=\max \left(\left|\sigma_{i j}^{(0)}\right|,\left|\sigma_{i j}^{(1)}\right|\right), \quad A_{i j}^{(r)}=\int_{\sigma_{i j}^{(0)}}^{\sigma_{i j}^{(1)}} \frac{(1+|h|)^{(n-r) / 2}}{\left(1-h^{2}\right)^{\hat{r} / 2}} d h .
$$

Then, for any $u_{1}, \ldots, u_{d}>0$, for some positive constant $C_{n, r}$ depending only on $n$ and $r$,

$$
\begin{aligned}
& \mathbb{P}\left(\bigcap_{i=1}^{d}\left\{\xi_{r: n}^{(0)}(i) \leq u_{i}\right\}\right)-\mathbb{P}\left(\bigcap_{i=1}^{d}\left\{\xi_{r: n}^{(1)}(i) \leq u_{i}\right\}\right) \\
& \leq C_{n, r} \sum_{1 \leq i<j \leq d}\left(u_{i}+u_{j}\right)^{-(n-r)}\left(A_{i j}^{(r)}\right)^{+} \exp \left(-\frac{\hat{r}\left(u_{i}^{2}+u_{j}^{2}\right)}{2\left(1+\rho_{i j}\right)}\right) .
\end{aligned}
$$

Lemma 5 Under the conditions of Theorem 2, for any $\varepsilon \in(0,1)$, there exist positive constants $K$ and $\rho$ depending only on $\varepsilon, \alpha$ and $\lambda$ such that

$$
\mathbb{P}\left(\sup _{S \leq t \leq T} \frac{X_{r: n}(t)}{f_{p}(t)} \leq 1\right) \leq \exp \left(-\frac{(1-\varepsilon)}{(1+\varepsilon)} \int_{S+1}^{T} \mathbb{P}\left(\sup _{t \in[0,1]} X_{r: n}(t)>f_{p}(u)\right) \mathrm{d} u\right)+K S^{-\rho}
$$

for any $T-1 \geq S \geq K$.

Proof Let, for any $i \geq 0$ and $\varepsilon \in(0,1)$,

$$
s_{i}=S+i(1+\varepsilon), \quad t_{i}=s_{i}+1, \quad x_{i}=f_{p}\left(t_{i}\right), \quad I_{i}=\left(s_{i}, t_{i}\right]
$$

For some $\theta>0$, define grid points in the interval $I_{i}$, as follows

$$
s_{i, u}=s_{i}+u q_{i}, \quad 0 \leq u \leq L_{i}, \quad L_{i}=\left[1 / q_{i}\right], \quad q_{i}=\theta x_{i}^{-\frac{2}{\alpha}} .
$$

Since $f_{p}$ is an increasing function, it easily follows that, with $T(S, \varepsilon)=[(T-S-$ $1) /(1+\varepsilon)]$,

$$
\begin{aligned}
\mathbb{P}\left(\sup _{S \leq t \leq T} \frac{X_{r: n}(t)}{f_{p}(t)} \leq 1\right) & \leq \mathbb{P}\left(\bigcap_{i=0}^{T(S, \varepsilon)}\left\{\sup _{t \in I_{i}} X_{r: n}(t) \leq x_{i}\right\}\right) \\
& \leq \mathbb{P}\left(\bigcap_{i=0}^{T(S, \varepsilon)}\left\{\max _{0 \leq u \leq L_{i}} X_{r: n}\left(s_{i, u}\right) \leq x_{i}\right\}\right)
\end{aligned}
$$


For any $1 \leq l \leq n$ and $i \geq 0$, let $X_{l, i}$ be an independent copy of the process $X_{l}$. Define a sequence of processes $Y_{l}=\left\{Y_{l}(t): t \in \cup_{i} I_{i}\right\}$ as $Y_{l}(t)=X_{l, i}(t)$, if $t \in I_{i}$. Let $Y_{r: n}=\left\{Y_{r: n}(t): t \geq 0\right\}$ be the $r$ th order statistic of $Y_{1}, \ldots, Y_{n}$. Put

$$
\begin{aligned}
\sigma_{i l, j k}^{(0)} & :=\mathbb{E} X_{l}(i) X_{k}(j)=r(|j-i|) 1_{\{l=k\}}=: \sigma_{i j}^{(0)} 1_{\{l=k\}}, \\
\sigma_{i l, j k}^{(1)}: & : \mathbb{E} Y_{l}(i) Y_{k}(j)=r(|j-i|) 1_{\{l=k\}} 1_{\left\{\exists m: i, j \in I_{m}\right\}}=: \sigma_{i j}^{(1)} 1_{\{l=k\}},
\end{aligned}
$$

and note that

$$
\begin{aligned}
\rho_{i j} & =\max \left(\left|\sigma_{i j}^{(0)}\right|,\left|\sigma_{i j}^{(1)}\right|\right)=|r(|j-i|)|, \\
A_{i j}^{(r)} & =\int_{\sigma_{i j}^{(0)}}^{\sigma_{i j}^{(1)}} \frac{(1+|h|)^{2(n-r)}}{\left(1-h^{2}\right)^{\hat{r} / 2}} \mathrm{~d} h=1_{\left\{\forall m: i, j \notin I_{m}\right\}} \int_{0}^{r(j-i)} \frac{(1+|h|)^{2(n-r)}}{\left(1-h^{2}\right)^{\hat{r} / 2}} \mathrm{~d} h \\
& =: 1_{\left\{\forall m: i, j \notin I_{m}\right\}}\left|\tilde{A}_{i j}^{(r)}\right| .
\end{aligned}
$$

Now using Lemma 4 we find that

$$
\begin{aligned}
& \mathbb{P}\left(\bigcap_{i=0}^{T(S, \varepsilon)}\left\{\max _{0 \leq u \leq L_{i}} X_{r: n}\left(s_{i, u}\right) \leq x_{i}\right\}\right) \\
& \leq \prod_{i=0}^{T(S, \varepsilon)} \mathbb{P}\left(\max _{0 \leq u \leq L_{i}} X_{r: n}\left(s_{i, u}\right) \leq x_{i}\right) \\
& +C_{n, r} \sum_{0 \leq i<j \leq T(S, \varepsilon)} \sum_{\substack{0 \leq u \leq L_{i} \\
0 \leq v \leq L_{j}}}\left(x_{i} x_{j}\right)^{-(n-r)}\left|\tilde{A}_{s_{i, u} s_{j, v}}^{(r)}\right| \exp \left(-\frac{\hat{r}\left(x_{i}^{2}+x_{j}^{2}\right)}{2\left(1+\left|r\left(s_{j, v}-s_{i, u}\right)\right|\right)}\right) \\
& =: P_{1}+P_{2} .
\end{aligned}
$$

\section{Estimate of $P_{1}$.}

Since $X_{r: n}$ is a stationary process, from Eq. 5 combined with Lemma 3, for any $\varepsilon \in(0,1)$, sufficiently large $\theta$ and $S$,

$$
\begin{aligned}
P_{1} & \leq \exp \left(-\sum_{i=0}^{T(S, \varepsilon)} \mathbb{P}\left(\max _{0 \leq u \leq L_{i}} X_{r: n}\left(s_{i, u}\right)>x_{i}\right)\right) \\
& \leq \exp \left(-(1-\varepsilon) \sum_{i=0}^{T(S, \varepsilon)} \mathbb{P}\left(\sup _{t \in[0,1]} X_{r: n}(t)>f_{p}\left(t_{i}\right)\right)\right) \\
& \leq \exp \left(-\frac{1-\varepsilon}{1+\varepsilon} \int_{S+1}^{T} \mathbb{P}\left(\sup _{t \in[0,1]} X_{r: n}(t)>f_{p}(u)\right) \mathrm{d} u\right) .
\end{aligned}
$$

Estimate of $P_{2}$. 
Noting that, for any $0 \leq i<j, 0 \leq u \leq L_{i}, 0 \leq v \leq L_{j}$;

$$
s_{j, v}-s_{i, u}=s_{j}+v q_{j}-s_{i}-u q_{i}=(j-i)(1+\varepsilon)+v q_{j}-u q_{i} \geq(j-i) \varepsilon,
$$

we have

$$
\sup _{\substack{0 \leq u \leq L_{i}, 0 \leq v \leq L_{j}}}\left|r\left(s_{j, v}-s_{i, u}\right)\right| \leq \sup _{\left|s-s^{\prime}\right| \geq(j-i) \varepsilon}\left|r\left(s-s^{\prime}\right)\right|=r^{*}((j-i) \varepsilon) \leq r^{*}(\varepsilon)<1
$$

Without loss of generality assume that $\lambda<2$. From (2) it follows that there is $s_{0}$ such that for every $s>s_{0}$,

$$
r^{*}(s) \leq s^{-\lambda} \leq \min (1, \lambda) / 4
$$

Finally, since the integrand in the definition of $\tilde{A}_{s_{i, u}}^{(r)} s_{j, v}$ is continuous and bounded on $\left[0, r^{*}(\varepsilon)\right]$, there exists a generic constant $K$ not depending on $S$ and $T$, which may differ from line to line, such that

$$
\left|\tilde{A}_{s_{i, u} s_{j, v}}^{(r)}\right| \leq K\left|r\left(s_{j, v}-s_{i, u}\right)\right| \leq K r^{*}((j-i) \varepsilon) .
$$

Therefore, for sufficiently large $S$,

$$
\begin{aligned}
& P_{2} \leq K \sum_{0 \leq i<j \leq T(S, \varepsilon)} L_{i} L_{j} r^{*}((j-i) \varepsilon) \exp \left(-\frac{\hat{r}\left(x_{i}^{2}+x_{j}^{2}\right)}{2\left(1+r^{*}((j-i) \varepsilon)\right.}\right) \\
& \leq K\left(\sum_{\substack{0<j-i \leq 2 s_{0} \\
0 \leq i<j \leq T(S, \varepsilon)}}+\sum_{\substack{j-i>2 s_{0} \\
0 \leq i<j \leq T(S, \varepsilon)}}\right) \\
& \leq K\left(\sum_{i=0}^{\infty} x_{i}^{\frac{4}{\alpha}} \exp \left(-\frac{\hat{r} x_{i}^{2}}{1+r^{*}(\varepsilon)}\right)\right. \\
& \left.+\sum_{\substack{j-i>2 s_{0} \\
0 \leq i<j \leq T(S, \varepsilon)}} x_{i}^{\frac{2}{\alpha}} x_{j}^{\frac{2}{\alpha}}(j-i)^{-\lambda} \exp \left(-\frac{\hat{r}\left(x_{i}^{2}+x_{j}^{2}\right)}{2\left(1+\frac{\lambda}{4}\right)}\right)\right) \\
& \leq K\left(\sum_{i=0}^{\infty} t_{i}^{-\frac{2}{1+\sqrt{r^{*}(\varepsilon)}}}+\sum_{\substack{j-i>2 s_{0} \\
0 \leq i<j \leq T(S, \varepsilon)}} t_{i}^{-\frac{1}{1+\frac{\lambda}{2}}} t_{j}^{-\frac{1}{1+\frac{\lambda}{2}}}(j-i)^{-\lambda}\right) .
\end{aligned}
$$

We can bound the first sum from the above by

$$
K \sum_{i=0}^{\infty}(S+i)^{-\frac{2}{1+\sqrt{r^{*}(\varepsilon)}}} \leq K S^{-\frac{1-\sqrt{r^{*}(\varepsilon)}}{4}}
$$


The second sum is bounded from above by

$$
\begin{aligned}
\sum_{S \leq i<j<\infty} & i^{-\frac{1}{1+\frac{\lambda}{2}}} j^{-\frac{1}{1+\frac{\lambda}{2}}}(j-i)^{-\lambda}=\sum_{j=S}^{\infty} j^{-\frac{1}{1+\frac{\lambda}{2}}} \sum_{i=S}^{j-1} i^{-\frac{1}{1+\frac{\lambda}{2}}}(j-i)^{-\lambda} \\
& \leq \sum_{j=S}^{\infty} j^{-\frac{1}{1+\frac{\lambda}{2}}}\left((j / 2)^{-\lambda} \sum_{i=S}^{[j / 2]} i^{-\frac{1}{1+\frac{\lambda}{2}}}+(j / 2)^{-\frac{1}{1+\frac{\lambda}{2}}} \sum_{i=[j / 2]}^{j-1}(j-i)^{-\lambda}\right) \\
& \leq K \sum_{j=S}^{\infty} j^{-\frac{1}{1+\frac{\lambda}{2}}}\left(j^{-\lambda+1-\frac{1}{1+\frac{\lambda}{2}}}+j^{-\frac{1}{1+\frac{\lambda}{2}}}\left(\log j \cdot 1_{\{\lambda \in[1,2)\}}+j^{-\lambda+1} 1_{\{\lambda \in(0,1)\}}\right)\right) \\
& \leq K\left(\sum_{j=S}^{\infty} j^{-\frac{2}{1+\frac{\lambda}{2}}} \log j \cdot 1_{\{\lambda \in[1,2)\}}+\sum_{j=S}^{\infty} j^{1-\lambda-\frac{2}{1+\frac{\lambda}{2}}} \cdot 1_{\{\lambda \in(0,1)\}}\right) \\
& \leq K\left(S^{1-\frac{2}{1+\frac{\lambda}{2}}} \log S \cdot 1_{\{\lambda \in[1,2)\}}+S^{2-\lambda-\frac{2}{1+\frac{\lambda}{2}}} \cdot 1_{\{\lambda \in(0,1)\}}\right) .
\end{aligned}
$$

Hence, for some positive constant $\rho$, depending only on $\varepsilon, \alpha$ and $\lambda$,

$$
P_{2} \leq K S^{-\rho}
$$

which finishes the proof.

Lemma 6 Under the conditions of Theorem 2, for any $\varepsilon \in(0,1)$, there exist positive constants $K$ and $\rho$ depending only on $\varepsilon, \alpha$ and $\lambda$ such that

$$
\begin{aligned}
& \mathbb{P}\left(\bigcap_{i=0}^{[T-S]}\left\{\max _{0 \leq u \leq\left[y_{i}^{\frac{2}{\alpha}} / \theta_{i}\right]} X_{r: n}\left(S+i+u \theta_{i} y_{i}^{-\frac{2}{\alpha}}\right) \leq y_{i}-\frac{\theta_{i}^{\alpha / 4}}{y_{i}}\right\}\right) \\
& \geq \frac{1}{4} \exp \left(-(1+\varepsilon) \int_{S}^{T} \mathbb{P}\left(\sup _{t \in[0,1]} X_{r: n}(t)>f_{p}(u)\right) \mathrm{d} u\right)-K S^{-\rho},
\end{aligned}
$$

for any $T-1 \geq S \geq K$, where $y_{i}=f_{p}(S+i)$ and $\theta_{i}=y_{i}^{-\frac{8}{\alpha}}$.

Proof Let, for any $i \geq 0, a_{i}=S+i$ so that $y_{i}=f_{p}\left(a_{i}\right)$. Define grid points in the interval $\left(a_{i}, a_{i+1}\right]$ as follows

$$
a_{i, u}=a_{i}+u q_{i}, \quad 0 \leq u \leq L_{i}, \quad L_{i}=\left[1 / q_{i}\right], \quad q_{i}=\theta_{i} y_{i}^{-\frac{2}{\alpha}}
$$


Finally, put $\hat{y}_{i}=y_{i}-\theta_{i}^{\frac{\alpha}{4}} / y_{i}$. Similarly as in the proof of Lemma 5, using Lemma 4 we have

$$
\begin{aligned}
& \mathbb{P}\left(\bigcap_{i=0}^{[T-S]}\left\{\max _{0 \leq u \leq L_{i}} X_{r: n}\left(a_{i, u}\right) \leq \hat{y}_{i}\right\}\right) \\
& \geq \prod_{i=0}^{[T-S]} \mathbb{P}\left(\max _{0 \leq u \leq L_{i}} X_{r: n}\left(a_{i, u}\right) \leq \hat{y}_{i}\right) \\
& \quad-C_{n, r} \sum_{0 \leq i<j \leq[T-S]} \sum_{\substack{0 \leq u \leq L_{i} \\
0 \leq v \leq L_{j}}}\left(\hat{y}_{i} \hat{y}_{j}\right)^{-(n-r)}\left(-\tilde{A}_{a_{i, u}(r) a_{j, v}}^{(r)}\right)^{+} \\
& \quad \exp \left(-\frac{\hat{r}\left(\hat{y}_{i}^{2}+\hat{y}_{j}^{2}\right)}{2\left(1+\left|r\left(a_{j, v}-a_{i, u}\right)\right|\right)}\right) \\
& =: \\
& P_{1}^{\prime}-P_{2}^{\prime},
\end{aligned}
$$

where $\tilde{A}_{a_{i, u} a_{j, v}}^{(r)}$ is as in (9).

Estimate of $P_{1}^{\prime}$.

Note that, by Lemma 3 combined with Eq. 5 ,

$$
\begin{aligned}
P_{1}^{\prime} & \geq \frac{1}{4} \exp \left(-\sum_{i=0}^{[T-S]} \mathbb{P}\left(\max _{0 \leq u \leq L_{i}} X_{r: n}\left(a_{i, u}\right)>\hat{y}_{i}\right)\right) \\
& \geq \frac{1}{4} \exp \left(-\sum_{i=0}^{[T-S]} \mathbb{P}\left(\sup _{t \in[0,1]} X_{r: n}(t)>\hat{y}_{i}\right)\right) \\
& \geq \frac{1}{4} \exp \left(-(1+\varepsilon) \sum_{i=0}^{[T-S]} \mathbb{P}\left(\sup _{t \in[0,1]} X_{r: n}(t)>y_{i}\right)\right) \\
& \geq \frac{1}{4} \exp \left(-(1+\varepsilon) \int_{S}^{T} \mathbb{P}\left(\sup _{t \in[0,1]} X_{r: n}(t)>f_{p}(u)\right) \mathrm{d} u\right),
\end{aligned}
$$

provided that $S$ is sufficiently large.

\section{Estimate of $P_{2}^{\prime}$.}

Noting that, for $j \geq i+2$, and any $0 \leq u \leq L_{i}, 0 \leq v \leq L_{j}$;

$$
a_{j, v}-a_{i, u}=a_{j}+v q_{j}-a_{i}-u q_{i} \geq j-i-1,
$$

we have

$$
\sup _{\substack{0 \leq u \leq L_{i} \\ 0 \leq v \leq L_{j}}}\left|r\left(a_{j, v}-a_{i, u}\right)\right| \leq \sup _{\left|s-s^{\prime}\right| \geq j-i-1}\left|r\left(s-s^{\prime}\right)\right|=r^{*}(j-i-1) \leq r^{*}(1)<1
$$


Since the integrand in definition of $\tilde{A}_{a_{i, u}}^{(r)} a_{j, v}$ is continuous and bounded on $\left[0, r^{*}(1)\right]$, there exists a constant $K$ such that

$$
\left|\tilde{A}_{a_{i, u} a_{j, v}}^{(r)}\right| \leq K r\left(a_{j, v}-a_{i, u}\right) \leq K r^{*}(j-i-1)<K
$$

On the other hand, by (1), there exist positive constants $s_{0}<1$, such that, for every $0 \leq s \leq s_{0}$,

$$
\tilde{A}_{0 s}^{(r)} \geq r(s) \geq 1-2|s|^{\alpha}>0 .
$$

Hence,

$$
\begin{aligned}
& \left(-\tilde{A}_{a_{i, u} a_{j, v}}^{(r)}\right)^{+}=0, \quad \text { if } j=i+1, \quad 1+v q_{j}-u q_{i} \leq s_{0}, \\
& \left|r\left(a_{j, v}-a_{i, u}\right)\right| \leq r^{*}\left(s_{0}\right)<1, \quad \text { if } \quad j=i+1, \quad 1+v q_{j}-u q_{i}>s_{0}
\end{aligned}
$$

Therefore, by (11)-(13) we obtain

$$
\begin{aligned}
P_{2}^{\prime} \leq & \sum_{\substack{0 \leq i \leq[T-S]-1 \\
j=i+1}} \sum_{\substack{0 \leq u \leq L_{i} \\
0 \leq v \leq L_{j}}} \frac{1}{\sqrt{1-r^{*}\left(s_{0}\right)}} \exp \left(-\frac{\hat{r}\left(\hat{y}_{i}^{2}+\hat{y}_{j}^{2}\right)}{2\left(1+r^{*}\left(s_{0}\right)\right)}\right) \\
& +\sum_{\substack{0 \leq i \leq[T-S]-2 \\
i+2 \leq j \leq[T-S]}} \sum_{\substack{0 \leq u \leq L_{i} \\
0 \leq v \leq L_{j}}} \frac{r^{*}(j-i-1)}{\sqrt{1-r^{*}(1)}} \exp \left(-\frac{\hat{r}\left(\hat{y}_{i}^{2}+\hat{y}_{j}^{2}\right)}{2\left(1+r^{*}(j-i-1)\right)}\right) .
\end{aligned}
$$

Completely similar to the estimation of $P_{2}$ in the proof of Lemma 5, we can arrive that there exist positive constants $K$ and $\rho$, independent of $S$ and $T$, such that, for sufficiently large $S$,

$$
P_{2}^{\prime} \leq K S^{-\rho}
$$

The following lemma is a straightforward modification of Lemmas 3.1 and 4.1 of [14] and [11, Lemma 1.4].

Lemma 7 If Theorem 1 is true under the additional condition that for large $t$,

$$
\frac{2}{\hat{r}} \log t \leq f^{2}(t) \leq \frac{3}{\hat{r}} \log t
$$

it is true without the additional condition. 


\section{Proofs of the Main Results}

Proof of Theorem 1 Note that the case $\mathscr{I}_{f}<\infty$ is straightforward and does not need any additional knowledge on process $X_{r: n}$ apart from the assumption of stationarity. Indeed, for sufficiently large $T$,

$$
\sum_{i=[T]+1}^{\infty} \mathbb{P}\left(\sup _{t \in[i, i+1]} X_{r: n}(t)>f(i)\right)=\sum_{i=[T]}^{\infty} \mathbb{P}\left(\sup _{t \in[0,1]} X_{r: n}(t)>f(i+1)\right) \leq \mathscr{I}_{f}<\infty
$$

and the Borel-Cantelli lemma completes this part of the proof since $f$ is an increasing function.

Now let $f$ be any increasing function such that $\mathscr{I}_{f} \equiv \infty$. With the same notation as in Lemma 5 with $f$ instead of $f_{p}$, we find that, for any $S>0$,

$$
\begin{aligned}
\mathbb{P}\left(X_{r: n}(s)>f(s) \text { i.o. }\right) & \geq \mathbb{P}\left(\left\{\sup _{t \in I_{i}} X_{r: n}(t)>x_{i}\right\} \text { i.o. }\right) \\
& \geq \mathbb{P}\left(\left\{\max _{1 \leq u \leq L_{i}} X_{r: n}\left(s_{i, u}\right)>x_{i}\right\} \text { i.o. }\right)
\end{aligned}
$$

where, recall, $s_{i, u}=S+i(1+\varepsilon)+u \theta x_{i}^{-2 / \alpha}, L_{i}=\left[1 /\left(\theta x_{i}^{-2 / \alpha}\right)\right], \theta, \varepsilon>0$. Furthermore, for sufficiently large $S$ and $\theta$, cf. estimation of $P_{1}$,

$$
\sum_{i=0}^{\infty} \mathbb{P}\left(\max _{1 \leq u \leq L_{i}} X_{r: n}\left(s_{i, u}\right)>x_{i}\right) \geq \frac{1-\varepsilon}{1+\varepsilon} \int_{S}^{\infty} \mathbb{P}\left(\sup _{t \in[0,1]} X_{r: n}(t)>f(u)\right) \mathrm{d} u=\infty
$$

Let $E_{i}=\left\{\max _{1 \leq u \leq L_{i}} X_{r: n}\left(s_{i, u}\right) \leq x_{i}\right\}$, and note that

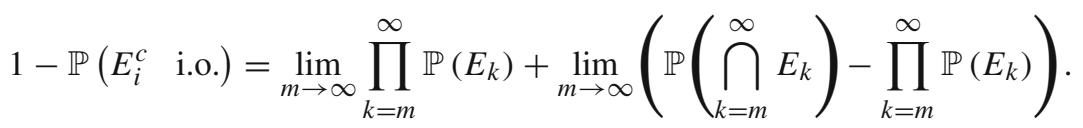

The first limit is zero as a consequence of (15), and the second limit will be zero because of the asymptotic independence of the events $E_{k}$. Indeed, there exist positive constants $K$ and $\rho$, such that for any $n>m$,

$$
A_{m, n}=\left|\mathbb{P}\left(\bigcap_{k=m}^{n} E_{k}\right)-\prod_{k=m}^{n} \mathbb{P}\left(E_{k}\right)\right| \leq K(S+m)^{-\rho},
$$

by the same calculations as in the estimate of $P_{2}$ in Lemma 5 after realizing that, by Lemma 7, we might restrict ourselves to the case when (14) holds. Therefore, $\mathbb{P}\left(E_{i}^{c}\right.$ i.o. $)=1$, which finishes the proof. 
Proof of Theorem 2 Step 1 . Let $p>1$, then, for every $\varepsilon \in\left(0, \frac{1}{4}\right)$,

$$
\liminf _{t \rightarrow \infty} \frac{\xi_{p}(t)-t}{h_{p}(t)} \geq-(1+2 \varepsilon)^{2} \text { a.s. }
$$

Proof Let $\left\{T_{k}: k \geq 1\right\}$ be a sequence such that $T_{k} \rightarrow \infty$, as $k \rightarrow \infty$. Put $S_{k}=$ $T_{k}-(1+2 \varepsilon)^{2} h_{p}\left(T_{k}\right)$. Then by Lemma 5 ,

$$
\begin{gathered}
\mathbb{P}\left(\frac{\xi_{p}\left(T_{k}\right)-T_{k}}{h_{p}\left(T_{k}\right)} \leq-(1+2 \varepsilon)^{2}\right)=\mathbb{P}\left(\xi_{p}\left(T_{k}\right) \leq S_{k}\right)=\mathbb{P}\left(\sup _{S_{k}<t \leq T_{k}} \frac{X_{r: n}(t)}{f_{p}(t)}<1\right) \\
\quad \leq \exp \left(-\frac{(1-\varepsilon)}{(1+\varepsilon)} \int_{S_{k}+1}^{T_{k}} \mathbb{P}\left(\sup _{t \in[0,1]} X_{r: n}(t)>f_{p}(u)\right) \mathrm{d} u\right)+2 K T_{k}^{-\rho},
\end{gathered}
$$

where the last inequality follows by the fact that $h_{p}(t)=o(t)$, so that $S_{k} \sim T_{k}$. Note that as $k \rightarrow \infty$

$$
\begin{aligned}
& \int_{S_{k}+1}^{T_{k}} \mathbb{P}\left(\sup _{t \in[0,1]} X_{r: n}(t)>f_{p}(u)\right) \mathrm{d} u \sim(1+2 \varepsilon)^{2} h_{p}\left(T_{k}\right) \mathbb{P}\left(\sup _{t \in[0,1]} X_{r: n}(t)>f_{p}\left(T_{k}\right)\right) \\
& =(1+2 \varepsilon)^{2} p \log _{2} T_{k} .
\end{aligned}
$$

Now take $T_{k}=\exp \left(k^{1 / p}\right)$. Then

$$
\sum_{k=0}^{\infty} \mathbb{P}\left(\xi_{p}\left(T_{k}\right) \leq S_{k}\right) \leq 2 K \sum_{k=0}^{\infty} k^{-(1+\varepsilon / 2)}<\infty
$$

Hence, by the Borel-Cantelli lemma,

$$
\liminf _{k \rightarrow \infty} \frac{\xi_{p}\left(T_{k}\right)-T_{k}}{h_{p}\left(T_{k}\right)} \geq-(1+2 \varepsilon)^{2} \quad \text { a.s. }
$$

Since $\xi(t)$ is a non-decreasing random function of $t$, for every $T_{k} \leq t \leq T_{k+1}$, we have

$$
\frac{\xi_{p}(t)-t}{h_{p}(t)} \geq \frac{h_{p}\left(T_{k}\right)}{h_{p}\left(T_{k+1}\right)} \frac{\xi_{p}\left(T_{k}\right)-T_{k+1}}{h_{p}\left(T_{k}\right)}=\frac{h_{p}\left(T_{k}\right)}{h_{p}\left(T_{k+1}\right)}\left(\frac{\xi_{p}\left(T_{k}\right)-T_{k}}{h_{p}\left(T_{k}\right)}-\frac{T_{k+1}-T_{k}}{h_{p}\left(T_{k}\right)}\right) .
$$

For $p>1$ elementary calculus implies

$$
\lim _{k \rightarrow \infty} \frac{T_{k+1}-T_{k}}{h_{p}\left(T_{k}\right)}=0, \quad \lim _{k \rightarrow \infty} \frac{h_{p}\left(T_{k}\right)}{h_{p}\left(T_{k+1}\right)}=1,
$$


so that

$$
\liminf _{t \rightarrow \infty} \frac{\xi_{p}(t)-t}{h_{p}(t)} \geq \liminf _{k \rightarrow \infty} \frac{\xi_{p}\left(T_{k}\right)-T_{k}}{h_{p}\left(T_{k}\right)} \text { a.s. }
$$

which finishes the proof of this step.

Step 2. Let $p>1$, then, for every $\varepsilon \in\left(0, \frac{1}{4}\right)$,

$$
\liminf _{t \rightarrow \infty} \frac{\xi_{p}(t)-t}{h_{p}(t)} \leq-(1-\varepsilon) \quad \text { a.s. }
$$

Proof As in the proof of the lower bound, put

$$
T_{k}=\exp \left(k^{\left(1+\varepsilon^{2}\right) / p}\right), \quad S_{k}=T_{k}-(1-\varepsilon) h_{p}\left(T_{k}\right), \quad k \geq 1 .
$$

Let

$$
B_{k}=\left\{\xi_{p}\left(T_{k}\right) \leq S_{k}\right\}=\left\{\sup _{S_{k}<s \leq T_{k}} \frac{X_{r: n}(s)}{f_{p}(s)}<1\right\} .
$$

It suffices to show $\mathbb{P}\left(B_{n}\right.$ i.o. $)=1$, that is

$$
\lim _{m \rightarrow \infty} \mathbb{P}\left(\bigcup_{k=m}^{\infty} B_{k}\right)=1
$$

Let $a_{i}^{k}=S_{k}+i$ and define grid points in the interval $\left[a_{i}^{k}, a_{i+1}^{k}\right]$ as follows

$$
\begin{aligned}
a_{i, u}^{k} & =a_{i}^{k}+u q_{i}^{k}, \quad 0 \leq u \leq L_{i}^{k}, \quad L_{i}^{k}=\left[1 / q_{i}^{k}\right], \quad q_{i}^{k}=\theta_{i}^{k}\left(y_{i}^{k}\right)^{-\frac{2}{\alpha}}, \quad \theta_{i}^{k}=\left(y_{i}^{k}\right)^{-\frac{8}{\alpha}}, \\
y_{i}^{k} & =f_{p}\left(a_{i}^{k}\right) .
\end{aligned}
$$

Put

$$
A_{k}=\bigcap_{i=0}^{\left[T_{k}-S_{k}\right]}\left\{\max _{0 \leq u \leq L_{i}^{k}} X_{r: n}\left(a_{i, u}^{k}\right) \leq y_{i}^{k}-\theta_{i}^{k} / y_{i}^{k}\right\} .
$$

Clearly, for $m \geq 1$,

$$
\mathbb{P}\left(\bigcup_{k=m}^{\infty} A_{k}\right) \leq \mathbb{P}\left(\bigcup_{k=m}^{\infty} B_{k}\right)+\sum_{k=m}^{\infty} \mathbb{P}\left(A_{k} \cap B_{k}^{c}\right)
$$


Put $\hat{y}_{i}^{k}=y_{i}^{k}-\theta_{i}^{k} / y_{i}^{k}$. Then, by Lemma 2, for some constants $K$ independent of $S$ and $T$, which may vary between (and among) lines,

$$
\begin{aligned}
\sum_{k=m}^{\infty} \mathbb{P}\left(A_{k} \cap B_{k}^{c}\right) & \leq \sum_{k=m}^{\infty} \sum_{i=0}^{\left[T_{k}-S_{k}\right]} \mathbb{P}\left(\max _{0 \leq u \leq L_{i}^{k}} X_{r: n}\left(a_{i, u}^{k}\right) \leq \hat{y}_{i}^{k}, \sup _{s \in[0,1]} X_{r: n}(s) \geq y_{i}^{k}\right) \\
& \leq K \sum_{k=m}^{\infty} \sum_{i=0}^{\infty}\left(y_{i}^{k}\right)^{\frac{2 \hat{r}}{\alpha}} \Psi\left(y_{i}^{k}\right)\left(\theta_{i}^{k}\right)^{\frac{\alpha}{2}-1} \Psi\left(K\left(\theta_{i}^{k}\right)^{-\frac{\alpha}{4}}\right) \\
& \leq K \sum_{k=m}^{\infty} \sum_{i=0}^{\infty}\left(a_{i}^{k} \log ^{1-p} a_{i}^{k}\right)^{-1}\left(\log a_{i}^{k}\right)^{\frac{4}{\alpha}-3 \alpha} \exp \left(-\frac{\log ^{2} a_{i}^{k}}{K}\right) \\
& \leq K \sum_{k=m}^{\infty} \sum_{i=0}^{\infty}\left(S_{k}+i\right)^{-3}\left(\log \left(S_{k}+i\right)\right)^{\frac{4}{\alpha}-3 \alpha+p-1} \\
& \leq K \sum_{k=m}^{\infty} S_{k}^{-1} \leq K m^{-4},
\end{aligned}
$$

provided $m$ is large enough. Therefore,

$$
\lim _{m \rightarrow \infty} \sum_{k=m}^{\infty} \mathbb{P}\left(A_{k} \cap B_{k}^{c}\right)=0
$$

and

$$
\lim _{m \rightarrow \infty} \mathbb{P}\left(\bigcup_{k=m}^{\infty} B_{k}\right) \geq \lim _{m \rightarrow \infty} \mathbb{P}\left(\bigcup_{k=m}^{\infty} A_{k}\right)
$$

To finish the proof of (18), we only need to show that

$$
\mathbb{P}\left(A_{n} \text { i.o. }\right)=1 \text {. }
$$

Similarly to (16), we have

$$
\int_{S_{k}}^{T_{k}} \mathbb{P}\left(\sup _{t \in[0,1]} X_{r: n}(t)>f_{p}(u)\right) \mathrm{d} u \sim(1-\varepsilon) p \log _{2} T_{k}
$$

Now from Lemma 6 it follows that

$$
\mathbb{P}\left(A_{k}\right) \geq \frac{1}{4} \exp \left(-\left(1-\varepsilon^{2}\right) p \log _{2} T_{k}\right)-K S_{k}^{-\rho} \geq \frac{1}{8} k^{-\left(1-\varepsilon^{4}\right)}
$$


for every $k$ sufficiently large. Hence,

$$
\sum_{k=1}^{\infty} \mathbb{P}\left(A_{k}\right)=\infty
$$

Applying Lemma 4, we get for $0 \leq t<k$

$$
\mathbb{P}\left(A_{k} A_{t}\right) \leq \mathbb{P}\left(A_{k}\right) \mathbb{P}\left(A_{t}\right)+M_{k, t}
$$

where, similarly to the proof of Lemma 5,

$$
M_{k, t}=C_{n, r} \sum_{\substack{\left.0 \leq i \leq T_{k}-S_{k}\right] \\ 0 \leq j \leq\left[T_{t}-S_{t}\right]}} \sum_{\substack{0 \leq u \leq L_{i}^{k} \\ 0 \leq v \leq L_{j}^{t}}}\left(\hat{y}_{i}^{k} \hat{y}_{j}^{t}\right)^{-(n-r)}\left|\tilde{A}_{s_{i, u}^{k} s_{j, v}^{t}}^{(r)}\right| \exp \left(-\frac{\hat{r}\left(\left(\hat{y}_{i}^{k}\right)^{2}+\left(\hat{y}_{j}^{t}\right)^{2}\right)}{2\left(1+\left|r\left(s_{i, u}^{k}-s_{j, v}^{t}\right)\right|\right)}\right),
$$

where

$$
\left|\tilde{A}_{s_{i, u}^{k} s_{j, v}^{t}}^{(r)}\right| \leq K\left|r\left(s_{i, u}^{k}-s_{j, v}^{t}\right)\right| .
$$

It is easy to see that,

$$
\frac{S_{k+1}-T_{k}}{T_{k+1}-T_{k}} \sim 1, \text { as } k \rightarrow \infty
$$

so that, for $0 \leq t<k$ and $k$ large enough, and assuming without loss of generality that $\lambda<2$,

$$
\begin{aligned}
\left|r\left(s_{i, u}^{k}-s_{j, v}^{t}\right)\right| & \leq r^{*}\left(S_{k}-T_{t}\right) \leq r^{*}\left(S_{k}-T_{k-1}\right) \leq K r^{*}\left(\frac{1}{2}\left(T_{k}-T_{k-1}\right)\right) \\
& \leq 2 K\left(T_{k}-T_{k-1}\right)^{-\lambda} \\
& \leq \min (1, \lambda) / 16 .
\end{aligned}
$$

Therefore,

$$
\begin{aligned}
M_{k, t} & \leq K\left(T_{k}-T_{k-1}\right)^{-\lambda} \sum_{\substack{0 \leq i \leq\left[T_{k}-S_{k}\right] \\
0 \leq j \leq\left[T_{t}-S_{t}\right]}} L_{i}^{k} L_{j}^{t} \exp \left(-\frac{\hat{r}\left(\left(\hat{y}_{i}^{k}\right)^{2}+\left(\hat{y}_{j}^{t}\right)^{2}\right)}{2\left(1+\frac{\lambda}{8}\right)}\right) \\
& \leq K\left(T_{k}-T_{k-1}\right)^{-\lambda} L_{\left[T_{k}-S_{k}\right]}^{k} L_{\left[T_{t}-S_{t}\right]}^{t} \sum_{\substack{0 \leq i \leq\left[T_{k}-S_{k}\right] \\
0 \leq j \leq\left[T_{t}-S_{t}\right]}}\left(a_{i}^{k}\right)^{-\frac{1}{1+\frac{\lambda}{4}}}\left(a_{j}^{t}\right)^{-\frac{1}{1+\frac{\lambda}{4}}}
\end{aligned}
$$




$$
\begin{aligned}
& \leq K\left(T_{k}-T_{k-1}\right)^{-\lambda} \log ^{\frac{5}{\alpha}} T_{k} \log ^{\frac{5}{\alpha}} T_{t} \cdot T_{k}^{\frac{\lambda}{4}} T_{t}^{\frac{\lambda}{4}} \\
& \leq K T_{k}^{-\frac{\lambda}{4}} \leq K \exp \left(-\lambda k^{\left(1+\varepsilon^{2}\right) / p} / 4\right) .
\end{aligned}
$$

Hence we have,

$$
\sum_{0 \leq t<k<\infty} M_{k, t}<\infty
$$

Now (19) follows from (21), (22) and (20) and the general form of the Borel-Cantelli lemma.

Step 3. If $p \in(0,1]$, then, for every $\varepsilon \in\left(0, \frac{1}{4}\right)$,

$$
\liminf _{t \rightarrow \infty} \frac{\log \left(\xi_{p}(t) / t\right)}{h_{p}(t) / t} \geq-(1+2 \varepsilon)^{2} \text { a.s. }
$$

and

$$
\liminf _{t \rightarrow \infty} \frac{\log \left(\xi_{p}(t) / t\right)}{h_{p}(t) / t} \leq-(1-\varepsilon) \quad \text { a.s. }
$$

Proof Put

$$
T_{k}=\exp \left(k^{1 / p}\right), \quad S_{k}=T_{k} \exp \left(-(1+2 \varepsilon)^{2} h_{p}\left(T_{k}\right)\right) .
$$

Proceeding the same as in the proof of (17), one can obtain that

$$
\liminf _{k \rightarrow \infty} \frac{\log \left(\xi_{p}\left(T_{k}\right) / T_{k}\right)}{h_{p}\left(T_{k}\right) / T_{k}} \geq-(1+2 \varepsilon)^{2} \text { a.s. }
$$

On the other hand it is clear that

$$
\liminf _{t \rightarrow \infty} \frac{\log \left(\xi_{p}(t) / t\right)}{h_{p}(t) / t} \geq \liminf _{k \rightarrow \infty} \frac{\log \left(\xi_{p}\left(T_{k}\right) / T_{k}\right)}{h_{p}\left(T_{k}\right) / T_{k}} \text { a.s. }
$$

since

$$
\lim _{k \rightarrow \infty} \frac{\log \left(T_{k} / T_{k+1}\right)}{h_{p}\left(T_{k}\right) / T_{k}}=0, \quad \lim _{k \rightarrow \infty} \frac{h_{p}\left(T_{k}\right)}{T_{k}} \frac{T_{k+1}}{h_{p}\left(T_{k+1}\right)}=1 .
$$

This proves (23).

Let

$$
T_{k}=\exp \left(k^{\left(1+\varepsilon^{2}\right) / p}\right), \quad S_{k}=T_{k} \exp \left(-(1-\varepsilon) h_{p}\left(T_{k}\right)\right)
$$

Noting that

$$
\frac{S_{k+1}-T_{k}}{S_{k+1}} \sim 1 \quad \text { as } k \rightarrow \infty
$$


along the same lines as in the proof of (18), we also have

$$
\liminf _{k \rightarrow \infty} \frac{\log \left(\xi_{p}\left(T_{k}\right) / T_{k}\right)}{h_{p}\left(T_{k}\right) / T_{k}} \leq-(1-\varepsilon) \text { a.s. }
$$

which proves (24).

Acknowledgements K. Dębicki was partially supported by National Science Centre Grant No. 2015/17/B/ ST1/01102 (2016-2019). Research of K. Kosiński was conducted under scientific Grant No. 2014/12/S/ST1/ 00491 funded by National Science Centre.

Open Access This article is distributed under the terms of the Creative Commons Attribution 4.0 International License (http://creativecommons.org/licenses/by/4.0/), which permits unrestricted use, distribution, and reproduction in any medium, provided you give appropriate credit to the original author(s) and the source, provide a link to the Creative Commons license, and indicate if changes were made.

\section{References}

1. Dębicki, K., Hashorva, E., Ji, L., Tabiś, K.: On the probability of conjunctions of stationary Gaussian process. Stat. Probab. Lett. 88, 141-148 (2014)

2. Dębicki, K., Hashorva, E., Ji, L., Ling, C.: Extremes of order statistics of stationary processes. Test 24, 229-248 (2015)

3. Dębicki, K., Hashorva, E., Ji, L., Tabiś, K.: Extremes of vector-valued Gaussian processes: exact asymptotics. Stoch. Process. Appl. 125, 4039-4065 (2015)

4. Dębicki, K., Hashorva, E., Ji, L., Ling, C.: Comparison inequalities for order statistics of Gaussian arrays (2016). arXiv:1503.09094v1

5. Hashorva, E., Ji, L., Piterbarg, V.I.: On the supremum of $\gamma$-reflected processes with fractional Brownian motion as input. Stoch. Process. Appl. 123, 4111-4127 (2013)

6. Hashorva, E., Korshunov, D., Piterbarg, V.I.: Asymptotic expansion of Gaussian chaos via probabilistic approach. Extremes 18(3), 315-347 (2015)

7. Leadbetter, M.R., Lindgren, G., Rootzen, H.: Extremes and Related Properties of Random Sequences and Processes. Springer, Berlin (1983)

8. Pickands III, J.: Upcrossing probabilities for stationary Gaussian processes. Trans. Am. Math. Soc. 145, 51-73 (1969)

9. Piterbarg, V.I.: Asymptotic Methods in the Theory of Gaussian Processes and Fields, Volume 148 of Translations of Mathematical Monographs. American Mathematical Society, Providence (1996)

10. Piterbarg, V.I., Prisyazhnyuk, V.: Asymptotic behavior of the probability of a large excursion for a nonstationary Gaussian processes. Theory Probab. Math. Stat. 18, 121-133 (1978)

11. Qualls, C., Watanabe, H.: An asymptotic $0-1$ behavior of Gaussian processes. Ann. Math. Stat. 42(6), 2029-2035 (1971)

12. Shao, Q.M.: An Erdös-Révész type law of the iterated logarithm for stationary Gaussian processes. Probab. Theory Relat. Fields 94, 119-133 (1992)

13. Spitzer, F.: Principles of Random Walk. Van Nostrand, Princeton (1964)

14. Watanabe, H.: An asymptotic property of Gaussian processes. Am. Math. Soc. 148(1), 233-248 (1970) 